\title{
A Study on the Integration Model of EIS Based on SOA
}

\author{
Xu Yang and Zhanhong Xin \\ School of Economics and Management, Beijing University of Posts and \\ Telecommunications, Beijing 100876, P.R. China yangx.china@gmail.com
}

\begin{abstract}
Recently, the enterprise application integration has been a crucial requirement for the progress of EIS. However, the integration of those heterogeneous or distributed information systems often encounters problems related to complex management and maintenance, as well as low compatibility. Nevertheless, to totally abandon the former system or to re-develop a new great EIS may result in costly investment, lengthy development cycle and tremendous risk accompanying with the rapid service changes. While, Serviceoriented architecture by adopting the loose coupling application program component technology, provides precisely defined and standardized service interface and reusable service that can assist the EIS engineers to dynamically construct the entire system more speedy, more reliable, and more reusable, and to cope with the demand changes more easily. This paper focuses on the integration model of EIS based on SOA. It proposes the implementation suggestions on SOA system and the rational risk analysis. Then, taking a telecommunication operator as an example, it presents a propositional framework for EIS integration based on SOA.
\end{abstract}

Keywords: $E I S, S O A, E A I$

\section{INTRODUCTION}

Nowadays, facing with the rapid change of market demand and the intensifying competition situation, the enterprises gradually shift their management pattern from product-oriented to customer-oriented. Therefore, the enterprises must build fluent information flow, break barriers within the enterprise information systems (EIS), eliminate information silos, and develop a holistic enterprise application platform. There is no wonder that enterprise application integration (EAI) tops the priority list of many CIOs [1]. The integration of enterprise IT system undergoes the following stages:

Stage 1: Integration of Isolated Systems. The integration is usually realized by direct access to data source. Due to the fact that the former information systems were developed to meet the specific demand of customers, the foundation of those IT systems were heterogeneous, distributed or isolated. Therefore, the need for specific program interfaces will result in tremendous numbers of interfaces, with expensive management and maintenance expenditure.

Stage 2: EAI. The enterprise starts to pay attention to the layout of EIS; EAI has occupied the main stream of integration. The usual way is to abandon the old application and to build a large complex enterprise application platform, which adopts 
ETL and Data Warehouse technique to integrate the old data, applies middleware (or integration adapter) to integrate the applications. This method realizes tight coupled structure, but results in low flexibility, complex structure and costly investment. What's more, this structure often implies huge risk accompanying with the rapid service changes, and long mature period accompanying with continuous update.

Stage 3: Service-Oriented EAI. Service-Oriented is regarded as the benchmark of EAI progress. The application systems could be invoked by other systems through uniform service interfaces. Meanwhile, the gradual maturity of Web Service provides the standard system architecture for integration, and meets the requirements on flexibility and interoperability, which together lead to a new stage of integration.

The paper discusses how SOA can be used to achieve EAI with loose coupling, high flexibility and making use of uniform infrastructures.

\section{INTEGRATION MODEL OF EIS BASED ON SOA}

Service-Oriented Architecture (SOA) is a component model, which connects the units with distinct application functions, as "Services", under the assistance of welldefined interfaces and contracts [2]. However, SOA is not a specific technique, but firm-level service-oriented system architecture. SOA enjoys the following characteristics and advantages:

1. Loosely coupled service architecture: SOA suffices the application environment with high agility and easy maintenance or correction. Those features establish the flexibility and diminish the complexity and dependency of services. Therefore, SOA can be chosen as the best IT architecture to satisfy the unknown future business demand.

2. Standardized uniform interface: SOA accomplishes the interaction between various services in a uniform and general way with the help of the neutral interface. In this way, both the location transparency of the application program and the independence of platform and protocol are realized, as well as the universal implement of SOA within firm or outside integration.

3. Combination and re-usage of services: SOA achieves dynamic and reusable service, and the by-products of this method are the protection of existing IT assets, the reduction of development and maintenance expenditure, the shortening of developing cycle, as well as the simplification of basic architecture and the lessening of risks.

\subsection{SOA Reference Architecture}

SOA is designed to be a holistic IT architecture, and the integration model based on SOA usually takes the following form, as shown in figure 1[3]. 


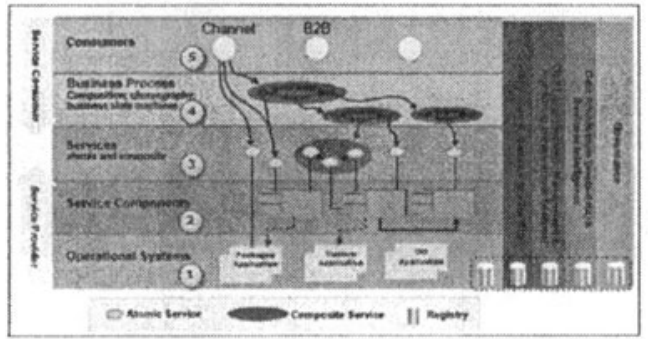

Figure 1. SOA Foundation Reference Architecture

This model consists of several layers. The lowest layer is the existing program or application software owned by the firms. The second one is called the Component Layer, which encapsulates the functions of the systems in the lowest layer into different service components. The third layer is the core one in SOA, which builds the function components of the lower layers into services with different functions. The forth one is called the Business Process Layer. Within this layer, the encapsulated services are used to construct the business process between systems. The fifth layer is viewed as the Expression Layer, which provides customers with interface services, such as portal. The five layers mentioned above require an integrated environment to support their operation, which is furnished by ESB (see the followings). Some complementary functions are also included in SOA systems, such as QoS, safety management, and so on.

Within the architecture of SOA, Enterprise Service Bus (ESB) plays a crucial role. ESB is a logical architecture, providing interconnection service such as Service Transform, Route, Notify and Augment between service consumer and service provider, as shown in figure 2. ESB simplifies the integration and flexible reuse of business components by providing a dependable and scalable infrastructure that connects disparate applications and IT resources, mediates their incompatibilities, orchestrates their interactions, and makes them broadly available as services for additional uses [4].

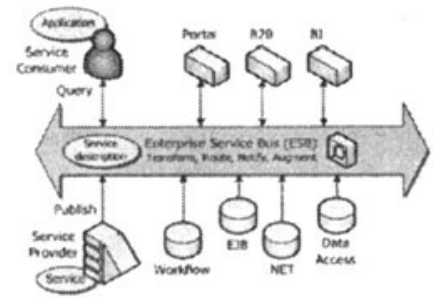

Figure 2. Enterprise Service Bus

\subsection{The Application of SOA}

SOA is related with the holistic IT architecture, which implies a lengthy time of construction. The way of top-down implementation is often accomplished with large 
scale of risks, including risks such as whether quick service response can be provided, whether security and QoS can be ensured, and whether lengthy deployment and large investment are needed, etc. Considering the agility of the services, the application of SOA could be based on the service-oriented modeling and architecture (SOMA [5]), start from the basic business demand, and adopt the bottom-up way of implementation, which has four stages:

In stage 1, construction of independent service: encapsulate the object-oriented applications into service functions, which are often the basic and reusable services or processes. In stage 2 , integration of services based on business functions: combine the encapsulated services using ESB, to realize the whole business process. In stage 3, firm-level IT transformation: transform the existing EIS, recombine business processes under service-oriented rules, and accomplish the EIS based on SOA. In stage 4, flexible business application according to demand: timely develop the future business process and application systems with the help of SOA. The application frame of SOA is shown in figure 3.

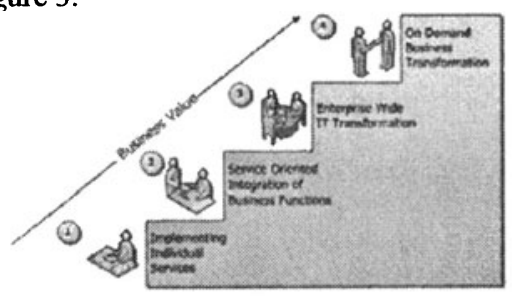

Figure 3. The Application Frame of SOA

\section{A CASE STUDY OF TELECOMMUNICATION OPERATOR}

\subsection{Problem Description}

The development of EIS of Chinese telecommunication operators has stepped into the integration stage. All of the Chinese telecom operators built large business support system (BSS, such as Charging and Settling System for telecommunication service), operation support system (OSS, such as Network Management System), management support system (MSS, such as OA, HR, Financial, etc. ). For example, through the centralization of multi-local-level systems into a single-province-level system, the business and operation support system (BOSS) of China Mobile has been a core business support platform involving functions such as mobile service commission and allocation, charging and settling, accounting, and so on. China Mobile also attempt to construct Uniform Data Platform and Integrated Application Platform to integrate data and application in firm-scope. However, lack of holistic plan, those systems still can not satisfy the development of telecom services and network.

Customer relationship management (CRM) is a representative system for this situation. Even though the management emphasis is shifting from service-centered to 
customer-centered, a uniform CRM application has not been formed at this moment. Due to the fact that the data is stored in different IT systems, there is a barrier for quick and accordant response when customers make a consultation, ask for a changing of service, or require other demand by different channels such as business hall, 10086(Call Center of China Mobile), 12580(Mobile Information Interactive Inquiry Center of China Mobile) or China Mobile website. The fail of quick response lowers the quality of service, prohibits the experience of customers, and results in the reduction of customer satisfaction, as shown in figure 4.

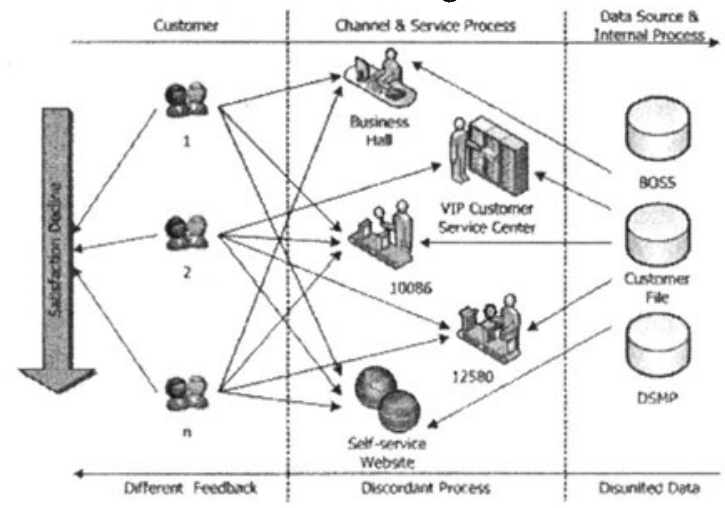

Figure 4. A CRM Lacking a Uniform Customer View

Under this condition, the operators launch on the construction of a holistic CRM system. Their primary consideration is to combine the services such as 10086,12580 into the BOSS system, to build Uniform Customer View and to realize the customeroriented service support system. However, the problems related to the large scale and heterogeneous of legacy systems would disadvantageously influence the holistic IT architecture and result in high expenditure.

\subsection{Solution of SOA}

To solve the above problems, a uniform CRM system could be build by SOA: first, componenting the inner-process functions (service commission and allocation, etc.) of BOSS or DSMP and the outer-process functions (customer inquiry, service alteration requisition, customer feedback, etc.) of 10086 or 12580 , and then encapsulate into reusable services, and in turn, combine a series of services into a complete customer serving process (e.g. service alteration process). This process can be adopted by the upper service systems, and finally the uniform CRM is realized. Because the uniqueness of services, the status of all services through all channels are identical from the perspective of customers, and thus, the Uniform Customer View is formulated. The transformation into customer-centered could be successfully completed through the integration of channels. The uniform CRM based on SOA is shown in figure 5 . 


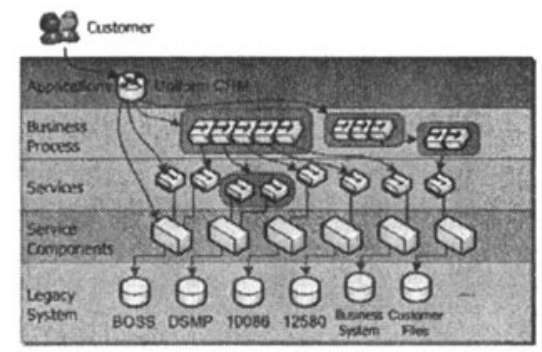

Figure 5. The Uniform CRM Based on SOA

\section{CONCLUSIONS}

Compared with other EIS architectures, the superiority of SOA is relying on flexibility, interoperability and reusability. By adopting loose coupling application program component model, it is possible to improve the value of business and the value of enterprises through analyzing and implementing of SOA.

For those telecom operators, who are experiencing transformation and facing with enormous subscribers and various telecom services with different tariffs and QoS, the deployment of SOA could integrate the former applications and provide powerful supports for BRP in one way of maintaining the existing flexible service applications and in the other way of constructing firm-level IT architecture to satisfy the future demand by quick response.

\section{ACKNOWLEDGEMENTS}

This work is supported by the Open Fund Project F0607-35, Key Laboratory of Information Management and Economics, MII, P.R.C.

\section{REFERENCES}

1. K.Channabasavaiah, K.Holley, and Jr.E.Tuggle, Migrating to a service-oriented architecture, IBM (2003). http://www.ibm.com/developerworks/library/ws-migratesoa/ (Accessed Apr. 2007).

2. Y. Liu and B. Li, The Operation Support Systems Based on SOA, Microcomputer Information. Volume 23, Number 1, pp.248-249, (2007).

3. Best Practices for SOA Management, IBM (2007). http://www.redbooks.ibm.com/abstracts/redp4233.pdf (Accessed Jun. 2007).

4. Anonymous, Enterprise Service Bus (ESB), Progress Software. http://www.sonicsoftware.com/solutions/service_oriented_architecture/enterprise service bus/index.ssp (Accessed Jun. 2007). 
5. N. Bieberstein, S. Bose, M. Fiammante, K. Jones, and R. Shah, Service-Oriented Architecture Compass: Business Value, Planning, and Enterprise Roadmap (IBM Press: 2005).

6. Web Services Architecture, W3C Working Draft (2002).

7. S. Pan, EAI and Its Application in Telecom Industry, Telecommunications Science. Number 7, pp.18-20, (2005).

8. S. Liu, W. Han, J. Liu, and G. Yin, Research on a Framework of Application Integration Based on SOA, Microelectronics \& Computer. Volume 23, pp.199-201, (2006). 